\title{
The Social Maze: A Collaborative Game to Motivate MS Patients for Upper Limb Training
}

\author{
Tom De Weyer ${ }^{1}$, Karel Robert ${ }^{1}$, Johanna Renny Octavia Hariandja ${ }^{1,2}$, \\ Geert Alders $^{3}$, and Karin Coninx ${ }^{1}$ \\ ${ }^{1}$ Hasselt University-tUL-IBBT, \\ Expertise Centre for Digital Media, \\ Diepenbeek, Belgium \\ ${ }^{2}$ Parahyangan Catholic University, Bandung, Indonesia \\ ${ }^{3}$ REVAL, PHL and BIOMED, \\ Hasselt University, Diepenbeek, Belgium \\ \{tom. deweyer, karel.robert, geert.alders, \\ karin.coninx\} @uhasselt.be, \\ johanna@unpar.ac.id
}

\begin{abstract}
Serious gaming is often used in the context of rehabilitation to increase the motivation of the patient to continue the rehabilitation program for a longer period. We investigate serious gaming in the context of rehabilitation programs for multiple sclerosis (MS) patients. Force feedback in combination with a virtual environment is used to establish a training environment supporting basic exercises and more advanced training games for the upper limb, in order to provide patients with an individual training program according to their capabilities. Based on our positive experiences with the use of individual games in this context, we explore "Social Gaming". More specifically, we designed and developed a "Social Maze", allowing patients to train together and to interact with the therapist. In this paper we clarify the game concept, setup and context in which the game is used, and report about the player experiences of patients and therapist.
\end{abstract}

Keywords: Rehabilitation, Serious Gaming, Social Gaming, Haptics.

\section{Introduction}

Social support can be beneficial for the engagement and motivation of patients to continue training. Patients' motivation during rehabilitation can be maintained and further enhanced through the incorporation of social interaction into the training exercises. Based on this belief, we have explored social rehabilitation training in a simple proof-of-concept game by designing and developing a simple collaborative game-like training exercise. The collaborative balance pump game is described in [1], which received the best paper award at GameDays 2010. An informal user study has revealed that most patients and therapists enjoyed training with this game. Inspired by 
this positive experience, we developed Social Maze, a more advanced collaborative training setup with a more elaborated game concept which optimally fits in our I-TRAVLE [2] training approach and system.

Before describing the Social Maze concept, we briefly describe the structured training approach as supported by the I-TRAVLE system. Robot-assisted rehabilitation and virtual environment (VE) technologies are combined in our I-TRAVLE system [3]. Using a haptic robot as the central hardware component, a software and hardware system setup has been realized to support systematic and personalized training for MS and stroke patients. The module for therapists allows us to define, to personalize, and to monitor the training. The patient interface gives access to training exercises and games using haptic feedback. In our structured training approach we provide basic training exercises which include only one skill component that has to be trained in the context of the upper limb rehabilitation (namely lifting, transporting, turning, pushing and reaching), but also more advanced training exercises or games which combine multiple skill components.

The Social Maze, the collaborative training game described in the next section, was designed to combine several skill components. Furthermore, the collaborative nature of the game brings in a social aspect and stimulates interaction between patients and therapists.

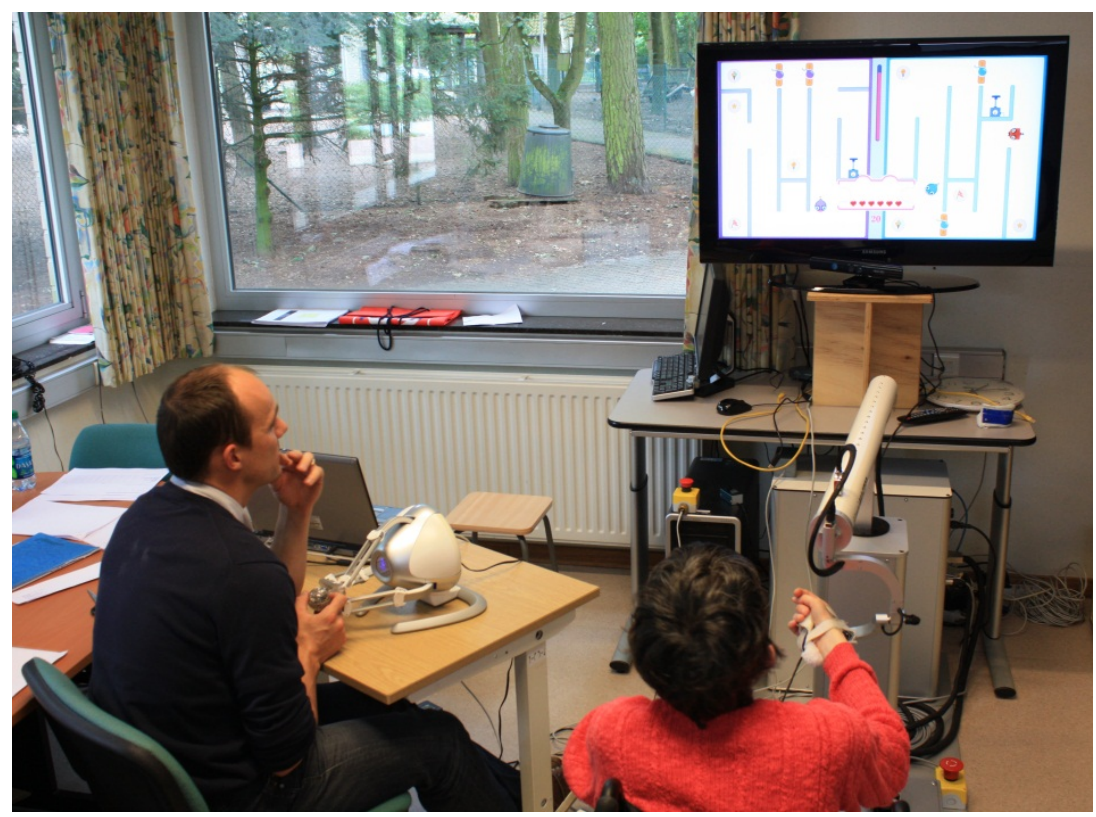

Fig. 1. System setup: with Novint falcon (therapist) and HapticMaster (patient) 


\section{Social Maze: The Game Concept}

The Social Maze is a collaborative game played by two persons. Several scenarios are possible: two patients playing together (empathetic scenario), a patient playing with a healthy relative or with a therapist (sympathetic scenario). It is exactly this last scenario which we have most extensively tested during the therapy sessions in the rehab center.

In all scenarios the patient will use the HapticMaster (Fig. 1) as the input device to manipulate an avatar. In the case of the empathetic scenario, a fellow patient will also use the HapticMaster as the input device. In the sympathetic scenario, the healthy player (e.g. a family member or therapist) can have different possibilities of input devices, they can either use the Novint Falcon, which is a consumer haptic input device, a Wiimote, a balance board or the Microsoft Kinect, a motion sensing input device which enables operations using body gestures.

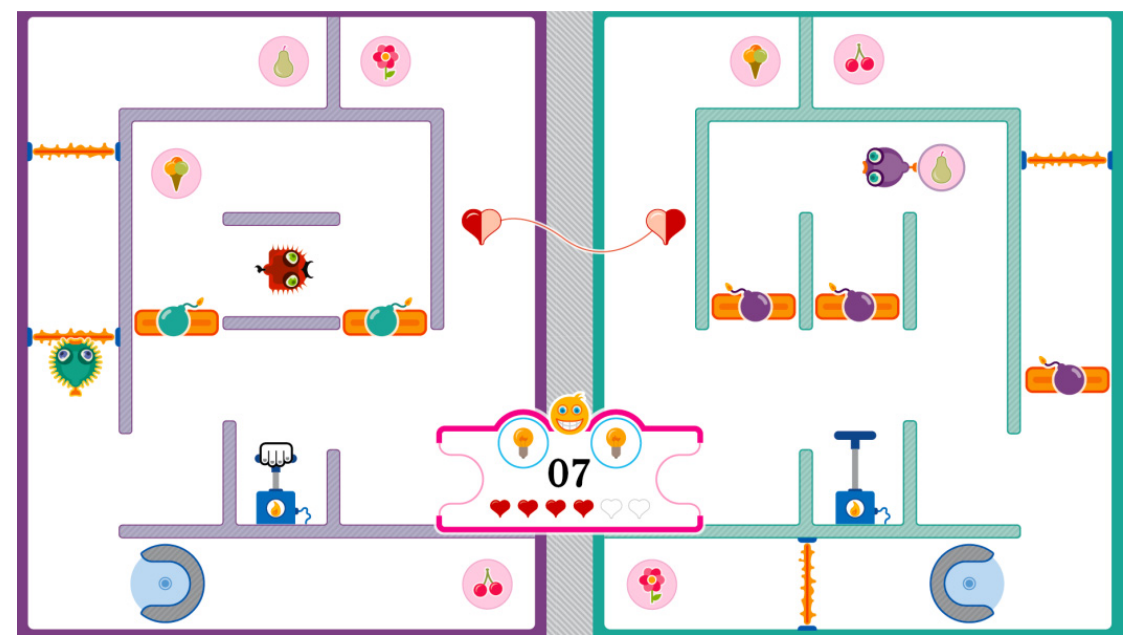

Fig. 2. Social Maze overview

Fig. 2 depicts our Social Maze with all game elements. The goal of this game is to collect all symbols, which represent points, by picking up each symbol and bringing it to the collecting bin. The elements of the game, with the pink background, were purposively designed so that two players (i.e. a patient and his/her training partner) have to collaborate as such to achieve the goal. Without collaboration, it is impossible to finish the game, so the game enforces so-called "closely-coupled collaboration".

The game area is divided into two. In each part the player, represented by a fishlike avatar (green for the left and purple for the right side), can move around his/her own maze to collect the symbols. Along the way there are some obstacles such as the laser beams that need to be avoided, the bombs that need to be demolished, the devil (red fish) that may not be encountered, and the rotators (with blue background) that need to be surmounted. Demolishing the bombs demands a tight collaboration 
between the players, where one player has to push the bomb trigger (push skill component) in order to destroy the bomb blocking the way of the other player. To pass the rotator, the players must enter it and perform the turning movement (turning skill component) to rotate it. When the players are hit by the laser beam or the devil, they will lose a life represented by a heart. To gain more lives, the players must attain the connecting heart (two connected half full hearts). Once the players pick up a symbol, they can place it in the collecting bin to earn points. After finishing collecting all symbols, a joint total score is shown to the players as depicted in the collective room.

The use of haptics makes sure that the patient's movement is kept within certain limits, for example it is not possible to go through the walls of the maze. Also, the haptic parameters allow us to control the complexity of the training depending on the physical performance of the patient, to make objects heavy or light etc., in order to strive for personalized training.

\section{Discussion and Conclusion}

The closely-coupled collaboration scenario as realized in the Social Maze turns out to be a successful (as shown in an RCT) and appreciated way to train. Besides providing the basic movements to be trained (skill components), the patient is immersed in a game concept that is challenging and stimulates social interaction. User tests, including observations, reveal that players discuss strategy and encourage each other. Pictures taken during the sessions show that social behavior is influenced by the game (e.g. mimicking each other's attitude). Patients and therapists also report more longterm social effects, such as teasing each other and referring back to gaming situations during previous sessions.

Acknowledgments. We acknowledge the INTERREG-IV program, project "Rehabilitation robotics 2", IVA-VLANED-1.14, Euregio Benelux, and the consortium partners.

See also www.i-travle.eu

\section{References}

1. Vanacken, L., Notelaers, S., Raymaekers, C., Coninx, K., van den Hoogen, W., Ijsselsteijn, W., Feys, P.: Game-Based Collaborative Training for Arm Rehabilitation of MS Pa-tients: A Proof-of-concept Game. In: Proceedings GameDays 2010, Darmstadt, Germany, pp. 65-75 (2010)

2. I-TRAVLE: Individualized, Technology-supported and Robot-Assisted Virtual Learning Environments, http: / /www.i-travle.eu

3. Feys, P., Alders, G., Gijbels, D., De Boeck, J., De Weyer, T., Coninx, K., Raymaekers, C., Truyens, V., Groenen, P., Meijer, K., Savelberg, H., Op, B.: Arm training in Multiple Sclerosis using Phantom: clinical relevance of robotic outcome measures. In: Proc. of ICORR 2009, pp. 576-581. IEEE (June 2009) ISBN 9781-4244-3789-4 